\title{
AN AUTOMATIC, INTERMITTENT ERUPTION, ARTIFICIAL
} GEYSER.

\section{By O. D. von ENGELN AND L. A. HAUSMAN, Geological Department, Physiography Laboratory, Cornell University.}

In the average high school the humble geography classroom, bare often of even a decent globe, can not begin to compete in attractiveness for students with the laboratories for chemistry and physics. In the chemistry laboratory the uninitiated student sees mysterious flasks and retorts, long rows of curiously labeled bottles of reagents; in the physics laboratory extensive equipment of complicated apparatus-all of which excite his interest. School boards, made up of prosaic business men, apparently respond to the same appeal, for it would seem that, presto, at the sound of the magic words, chemistry and physics, the public purse is opened quite freely to supply new complications. On the other hand, too scant attention is accorded requests for such commonplaces as maps and lantern slides for geography teaching. Perhaps this is a reflection of the attitude recorded recently by the business men of St. Louis who solemnly opined that geography had little, if any, direct vocational value. If that is true, one may also ask of what direct vocational value is a knowledge of the time honored $\mathrm{H}_{2} \mathrm{SO}_{4}$ to the average student. The real difficulty appears to be that geography as taught is entirely too practical to be popular.

Possibly if geography can be made a bit more spectactiar, pupils and school boards will be more alive to its merits. Visualizing the subject by means of lantern slides is a step in the right direction and serves especially in teaching the facts of general geography. Many of the topics in the physical geography courses usually given in high schools can also be illustrated with lantern slides to good advantage, especially in view of the tendency at present to put the enphasis on teaching the relation of the environment to the character of the human occupation of a region. But the origin and development of land forms, mountain and valley, plateau and delta, as well as of natural phenomena such as waterfalls, volcanoes, and geysers continue to comprise a large part of the study.

Nor is this unwise from either a practical or culturâl point of view. Certainly it is worth while to have a clear understanding of how the region in which one lives or to which one journeys 
got its form and features. In any event, such knowledge can be applied a hundred times by the average person before a need arises for an appreciation of $\mathrm{H}_{2} \mathrm{SO}_{4}$. It is when one travels in the West that one comes to understand, however, how uninformed most people are about natural phenomena. At the Grand Canyon and in the Yellowstone Park one may listen any day to a variety of vague guesses and marvelous explanations of the wonders to be seen in those regions. Accordingly, we can not be remiss in urging that physiography be taught, and taught interestingly enough to attract numbers of students.

So much by way of introduction. The particular purpose of this paper is to give directions for the construction of a model geyser that is both easy to set up and truly simulates the conditions attending the occurrence and eruptions of its natural counterpart. The introduction of this and other like illustrations as classroom experiments will do much to give zest to geographic study. The great difficulty with such attempts usually is that they are not truly representative, that they often fail to fulfil some essential condition of the natural phenomenon. Hence the net result is apt to be a misconception on the part of the students of some phase of the real situation.

This difficulty we think has been overcome in the present instance. In order to make it clear how the apparatus described is conceived to illustrate geyser eruptions, it is first necessary to review the chief facts about geysers. Geysers occur only in Iceland, New Zealand, and the Yellowstone Park. These three regions are at present or have in the recent geological past been the scene of great volcanic activity with attendant flows of lava thousands of feet thick. This lava has cooled at the surface to hard rock, but at no very great depth are hundreds, perhaps thousands, of feet thickness of still very hot rock. This because the very slow conduction of heat by rock permits of only very slow escape of heat from the buried masses, through the cooled outer crust. All known geyser occurrences are along the shores of streams or lakes; in other words, at valley bottoms-places where water seeping into the earth over highland areas normally again comes to the surface in springs. That is, rain and snowfall waters from plateau and mountain areas percolating through the rocks below commonly reappear at the surface in valley bottom areas. In the geyser regions the cool descending waters penetrate to the incandescent lavas through. multitudinous minute pores and fissures and then ascend through larger openings, the 
geyser and hot spring tubes, to the surface as hot water and steam. Gravity is responsible for the downward motion of the water; gravity (the pressure head of the descending films of water), plus the expansive energy developed by the heating in contact with the hot lava, accounts for the upward motion and reappearance of the water in hot springs and geysers. It is not essential that all the descending water come in contact with the hot lavas. Part of it may be converted into steam of such high temperature as to heat effectively a considerable volume of water that does not penetrate to great depths. The question whether the water will reappear as a geyser with periodic eruptions or as a hot spring with relatively quiet constant ebullition seems to depend on two factors- $(a)$ the size of the opening leading to the surface, $(b)$ its depth. If the opening is large in comparison to the supply of water and heat energy and if the tube is not of great depth, a hot spring results. On the other hand, a deep tube with high heat at the bottom and a free inflow of water through its sides will favor the geyser phenomenon. As the development of the geyser and hot spring tubes is at least in part due to the solvent action of the hot ascending waters, and to the explosive force of eruptions in the case of geysers, it follows that with increase in the diameter of an opening, a geyser may become a hot spring; by increase in depth of the tube, a hot spring may develop geyser characteristics. As a matter of fact, there are all gradations between the two forms in the Yellowstone Park.

Increase in depth of the tube tends to the development of geyser eruption because the greater the pressure, the higher the temperature necessary to make water boil, i. e., to convert it into steam with much greater volume. Thus while water boils at $212^{\circ} \mathrm{F}$. at sea level under atmospheric pressure, it must be heated to a temperature of $293^{\circ} \mathrm{F}$. before it can change to steam when weighed down by a column of water 100 feet high. Since the greatest heat is probably most commonly supplied at the bottom of the geyser tubes, it follows that, relatively, very high temperatures must be attained before the water there reaches the boiling point. This may be a very important condition for while the water at the bottom is heating, there is time for the rest of the tube to fill up and develop the requisite pressure. In fact, if the supply of heat is very great in proportion to the supply of water, a constantly erupting steam jet results; e. g., the Black Growler of the Yellowstone. It is also important to note that, coupled with 
the fact that they open to the surface at higher elevations, the fineness of the rock pores through which the cold water descends prevents their utilization as a path for the rapid geyser expulsions.

A geyser tube accordingly fills up with cold water by percolation through many small fissures. Eventually (the length of time required varying with the volume of water to be heated and the magnitude and temperature of the heat source), the bottom waters in the tube are heated to their high boiling point. A portion of their mass changes to steam with very much greater volume. This lifts all the column of water above. The water at the top of the tube boils over. This relieves the pressure throughout the whole column of water. Hence the water at many points in the lower part of the tube is suddenly enabled to boil, changes to steam, and the expansion of this forces the whole column of water out of the tube and the geyser erupts a mingled mass of water and steam. The eruption begins with a quiet outflow of water, the main outburst is mingled water and steam, the end is a hoarse roar of steam.

The essentials, then, to be provided for a model geyser, faithful in its construction and mechanism to the natural phenomenon, are (a) a source of cold water equivalent to the precipitation supplies; $(b)$ a method of admitting the cold water supply to the geyser tube with relative slowness, both to simulate the slow percolation through rock pores and fissures and to prevent a back flow of the heated waters; $(c)$ a deep-seated source of heat; $(d)$ a relatively open geyser tube; $(e)$ with a somewhat constructive outlet to differentiate it from a hot spring. These are all supplied in the apparatus figured and described below. The point $(b)$ is the particular new thing about this apparatus. Other descriptions of model geysers have appeared recently in the Scientific American Supplement, Septembe. 23,1911 , and in the Scientific American, December 19, 1914; also in the Encyclopedia Britannica, 11th Ed., article on "Geysers." The apparatus described is, however, complicated or not directly repesentative of the natural conditions.

The diagram, Figure 1, is practically self-explanatory. The construction in the tube from the reservoir is made by drawing out a glass tube at one point as shown in Figure 2. The rubber supply tube is cut to permit the insertion of the glass tube construction. Iron stands are used to support the reservoir and the geyser tube. The one supporting the reservoir is made high enough by slipping a section of pipe over the upright stem of the 
stand. To this pipe the neck of the bottle is lashed with soft copper wire. Note that the glass tube from the supply reservoir does not penetrate the flask so far as the one leading to the geyser tube. The water level in the flask when boiling begins will be below the supply tube outlet. The two-hole rubber stopper of the flask should be pressed in firmly. The diameter of the nozzle of the geyser tube should be of slightly greater diameter than the diameter of the constriction in the supply tube. The Bunsen burner flame should be quite high and hot.

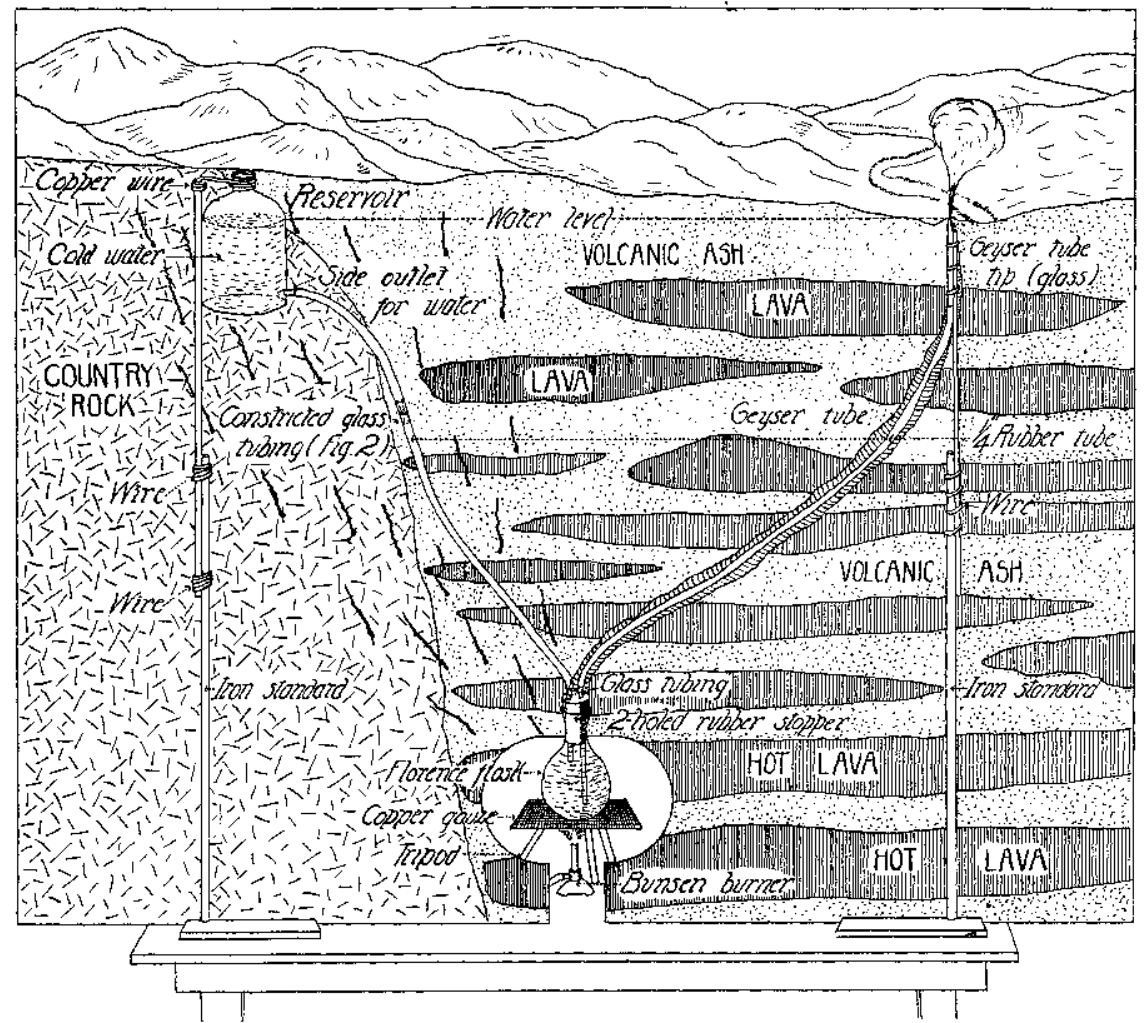

If the equal water levels in the reservoir and geyser tube before the beginning of an eruption are so adjusted that the water rises to within about one inch from the top of the geyser tube, eruptions proceed at regular intervals of about two minutes after the manner of Old Faithful which erupts once every hour. The model geyser as illustrated throws a stream about two feet high at the climax of the outburst as illustrated in the photograph, Figure 3. Preceding the eruption there is a quiet outflow of 
water for a second; at the end comes a jet of steam. After an eruption the flask slowly fills from the reservoir and the phenomena are automatically repeated. The reservoir should be quite

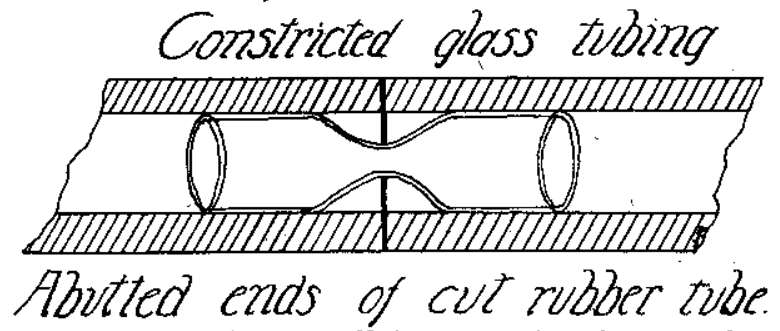

large in order to provide a sufficient supply of water for a number of eruptions in succession without lowering the water level too greatly (this increases the violence of the eruption) and also

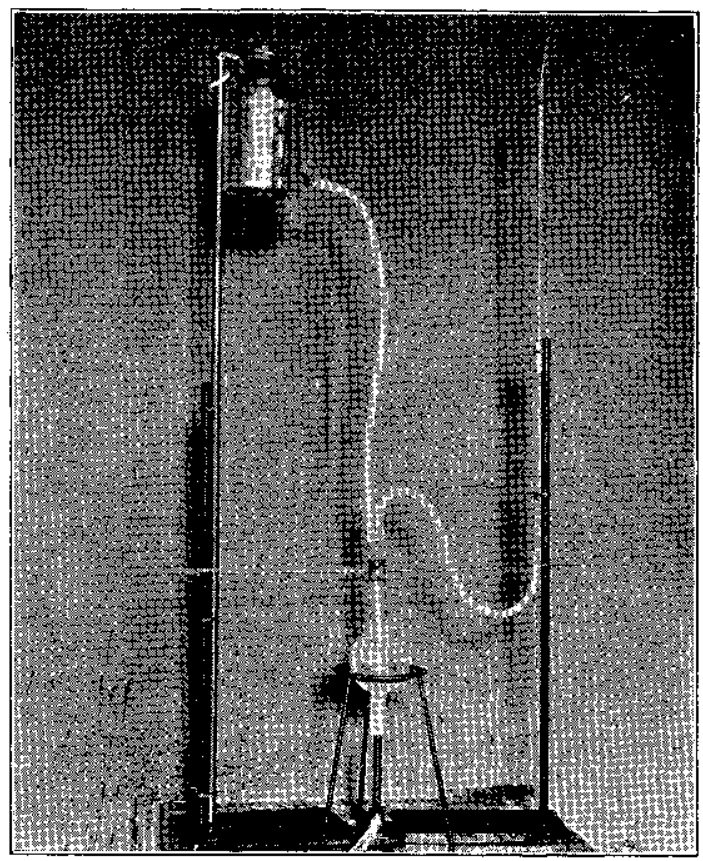

to prevent appreciable heating of the reserve water by heat conduction from the flask, as this diminishes the interval between eruptions. Ice fragments may be introduced to counteract such a tendency; these will represent the cold ice and snow waters from the mountain supply areas.

In order to make the experiment more realistic for classroom use, it is suggested that a cross-section drawing be made on a 
large sheet of cardboard and introduced as a background about the apparatus as illustrated in Figure 1. This will enable students to grasp at once the function of each portion of the mechanism and its relation to the natural conditions. The teacher will find two illustrated pamphlets entitled Geysers, by W. H. Weed, and Geological History of the Yellowstone National Park, by A. Hague, obtainable for ten cents each from the Superintendent of Documents, Washington, D. C., very helpful in connection with a study of geysers.

While geysers in themselves are perhaps the least significant among geographic phenomena, they are on the other hand only second to volcanic outbursts in spectacular features. Moreover, if properly interpreted, as with this model geyser, they serve to give students a clear insight of the nature of subterranean conditions and their relations to surface features. It is hoped in succeeding papers to suggest other experiments that will help to a better understanding of physiographic phenomena, and incidentally to liven up the course in geography.

\section{THE USE OF SODIUM SALTS IN PURIFYING AND CASTING CLAYS.}

In Technologic Paper, No. 5I, just issued by the Bureau of Standards, Department of Commerce, the results of some work involving the addition of small quantities of sodium carbonate and sodium silicate to clay suspensions are published. Clays, mixed with water, may be considered to consist of a system of fine particles varying from comparatively coarse to very fine grains, $0.003 \mathrm{~mm}$. and less in diameter. It is a curious fact that these fine particles are very sensitive to the influence of small quantities of alkalies, salts and acids, and hence the properties of the clays as a whole are affected to an important degree by such additions. Alkalies tend to break down the grains into still finer particles and to hold them in suspension. At the same time the fluidity of the system is increased so that a separation is possible between the particles capable of greatest dispersion (the clay substance proper) and the coarse particles which comprise the principal impurities. A method of purifying clays is thus suggested. Acids and salts act in the opposite sense, tending to coagulate the particles and to precipitate them. In the casting process, employed in the manufacture of sanitary ware and other clay products, the effect of the alkalies consists essentially in reducing the water content and with it the drying shrinkage, which is essential in preventing the cracking of the ware.

The characteristic effect of the alkalies upon different American clays was studied from several standpoints, particularly with reference to the viscosity of the clay suspensions, the absorption of the reagents, the strength of the treated mixture in the dried state, and the influence of time. The effect of different clays upon each other was also studied.

This publication is for free distribution and copies may be obtained by those interested upon request of the Bureau of Standards, Washington, D. C, 